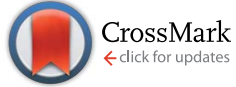

Cite this: Soft Matter, 2015, 11, 2479

Received 17th October 2014 Accepted 19th January 2015

DOI: $10.1039 / c 4 s m 02289 k$

www.rsc.org/softmatter

\section{Sorting of integral membrane proteins mediated by curvature-dependent protein-lipid bilayer interaction}

\begin{abstract}
Bojan Božič, ${ }^{a}$ Sovan L. Das ${ }^{b}$ and Saša Svetina*ac
Cell membrane proteins, both bound and integral, are known to preferentially accumulate at membrane locations with curvatures favorable to their shape. This is mainly due to the curvature dependent interaction between membrane proteins and their lipid environment. Here, we analyze the effects of the protein-lipid bilayer interaction energy due to mismatch between the protein shape and the principal curvatures of the surrounding bilayer. The role of different macroscopic parameters that define the interaction energy term is elucidated in relation to recent experiment in which the lateral distribution of a membrane embedded protein potassium channel KvAP is measured on a giant unilamellar lipid vesicle (reservoir) and a narrow tubular extension - a tether - kept at constant length. The dependence of the sorting ratio, defined as the ratio between the areal density of the protein on the tether and on the vesicle, on the inverse tether radius is influenced by the strength of the interaction, the intrinsic shape of the membrane embedded protein, and its abundance in the reservoir. It is described how the values of these constants can be extracted from experiments. The intrinsic principal curvatures of a protein are related to the tether radius at which the sorting ratio attains its maximum value. The estimate of the principal intrinsic curvature of the protein KvAP, obtained by comparing the experimental and theoretical sorting behavior, is consistent with the available information on its structure.
\end{abstract}

\section{Introduction}

"Membrane curvature is a vital property of cells: many cellular processes, such as cell migration, cell division, or endocytosis crucially depend on membrane remodeling reactions, but the understanding how cells change the shape of their membranes is still at an early stage". ${ }^{1}$ The molecular basis of membrane curvature control resides in integral membrane and membrane bound proteins that are sensitive to membrane curvature, ${ }^{2}$ as their energy and conformational state depend on the surrounding membrane curvature. ${ }^{3}$ If mobile, such proteins distribute on membranes with variable curvatures in an inhomogeneous manner. ${ }^{4}$

In a recent experiment, ${ }^{5}$ the membrane curvature dependent lateral distribution of the integral membrane protein potassium channel KvAP has been studied quantitatively. A biophysical system has been used, in which a narrow membrane tube, the tether, is pulled by optical tweezers out of an aspirated giant unilamellar vesicle (GUV) and kept at a constant length (Fig. 1a). Tether radius has been established according to the

${ }^{a}$ Institute of Biophysics, Faculty of Medicine, University of Ljubljana, Ljubljana, Slovenia. E-mail: sasa.svetina@mf.uni-lj.si

${ }^{b}$ Department of Mechanical Engineering, Indian Institute of Technology, Kanpur 208016, India

'Jožef Stefan Institute, Ljubljana, Slovenia requirements of the mechanical equilibrium and monitored by changing the aspiration pressure and consequently, membrane lateral tension. In this system, there are essentially two spatially separated regions of significantly different curvatures, a highly curved tether and a practically flat GUV. The corresponding protein densities and their ratio have been measured using the intensities of fluorescent dye molecules to tag the proteins. Further, by measuring the density of lipids, the tether radius, and hence the curvature, was determined independently. The key result of the study was the measured dependence of the sorting ratio, i.e., the ratio between the KvAP density (protein per lipid) on the tether and the GUV, on the tether curvature. KvAP density on the highly curved membrane of the tether was larger than on the vesicle main body. The similar experimental technique was also employed in the studies of curvature sensitivity of different BAR proteins. ${ }^{6-8}$

The experimental observations on the curvature dependent lateral sorting of membrane proteins can be interpreted by taking into consideration the effect of membrane proteins on membrane spontaneous curvature. ${ }^{9}$ The corresponding models are conceptually simple, and their variants are often used in interpretations of membrane curvature establishment and sensing. Aimon and coworkers ${ }^{5}$ interpreted their main result on the basis of a similar model proposed by Markin. ${ }^{\mathbf{1 0}}$ In this model, it was asserted that proteins because of their intrinsic shape cause a modification of membrane bending constant and 
(a)

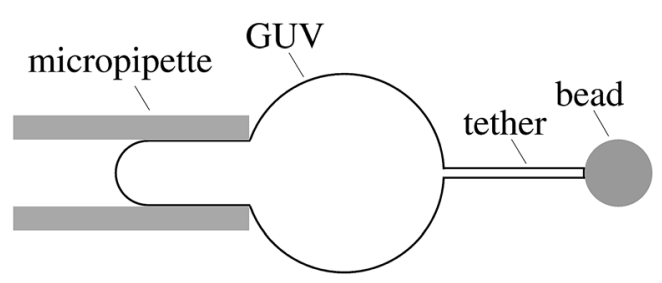

(b)

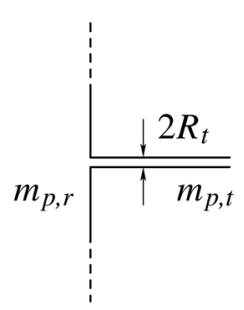

Fig. 1 Schematic representation of the treated model. (a) Experimental setup of a tether pulled out of a GUV aspirated into micropipette. (b) The substitute system under study involves some simplifying assumptions. The main body of the GUV is represented by a large flat surface, and the tether is represented by a cylinder of the radius $R_{t}$. The protein number densities on the large flat surface and on the cylinder are denoted by $m_{p, r}$ and $m_{p, t}$, respectively.

spontaneous curvature. The redistribution of proteins was explained by spatial variation of protein density yielding the match between local membrane spontaneous curvature and local membrane curvature, which gives rise to the minimum of the total membrane bending energy.

An alternative approach to study the effects of the proteinlipid bilayer interaction on the behavior of membranes with embedded proteins is to introduce a term for the strength of interactions between the protein and the membrane, which is a function of curvature. ${ }^{11-13}$ The parameters of this interaction term are defined in a general way so that they may represent different underlying physical or chemical mechanisms giving rise to the curvature dependent protein-lipid bilayer interaction. Few such mechanisms are, for example, the perturbed bending energy of the surrounding bilayer, ${ }^{3}$ locally modified lipid composition in multicomponent lipid bilayers, ${ }^{14}$ or the protein elasticity. ${ }^{15}$ The macroscopic parameters of the proteinlipid bilayer interaction can be viewed as a possible link between the microscopic and macroscopic properties of membranes. On one hand, the determination of their magnitude could be the target of molecular simulations on how curvature is sensed or generated by proteins, ${ }^{16,17}$ while on the other hand it is of interest to reveal in what way they define membrane macroscopic behavior. It has been shown that the approach to be employed here is more general than the spontaneous curvature model because the latter represents its limit at small interaction constants. ${ }^{12,13}$

This study will focus on the interaction of an embedded membrane protein with the surrounding lipid moiety expressed as the contribution to the membrane energy. We shall assume that proteins are rigid and mobile, and that they behave like a two-dimensional gas. By assuming that the protein is rigid, we are neglecting its internal degrees of freedom. In this limit, the sensing of membrane curvature is manifested by the lateral occupancy of proteins being enhanced in membrane regions that are favorable to their intrinsic shape and diminished in regions of unfavorable shape. We shall investigate how these parameters are manifested in the tether pulling experiment of protein curvature sensing. ${ }^{5}$ In view of the promising perspective of this experiment in the characterization of curvature forming and sensing properties of membrane proteins, we shall perform a detailed analysis of the information on the protein-lipid bilayer interaction that can be obtained from the determination of the dependence of the sorting ratio on the tether curvature. The analysis will be guided by the observation on the transmembrane protein $\mathrm{KvAP}^{5}$ but is meant to be valid also for other proteins. We will draw some conclusions about the underlying structural features of KvAP.

\section{The analysis}

We shall first define the energy terms that constitute the free energy of the treated system. In the next two subsections, we shall reveal the constants that define the protein density ratio in the case that all proteins have the same transmembrane orientation, first in the limit of low protein density and then for their finite density. In a separate subsection, we shall show the consequences of a bidirectional orientation of a given protein. In the last subsection, it will be shown how the constants that define the protein density ratio relate to the parameters of the protein-lipid bilayer interaction term.

\section{(i) Free energy of membrane embedded proteins}

The energy contribution due to protein-lipid bilayer interaction is, in the limit of rigid protein surface, the consequence of the mismatch between local membrane principal curvatures and the intrinsic principal curvatures of the protein. ${ }^{11,12}$ The intrinsic principal curvatures of the protein refer to the shape of the part of the protein which is in contact with the lipid bilayer. The general expression for the corresponding energy term has been derived ${ }^{18}$ and can be written as

$$
E=\frac{\kappa}{2}\left(H-H_{\mathrm{m}}\right)^{2}+\frac{\kappa^{*}}{2}\left[\Delta H^{2}-2 \Delta H \Delta H_{\mathrm{m}} \cos (2 \omega)+\Delta H_{\mathrm{m}}{ }^{2}\right]
$$

where $H=\left(C_{1}+C_{2}\right) / 2$ is the mean of the membrane principal curvatures $C_{1}$ and $C_{2}, \Delta H=\left(C_{1}-C_{2}\right) / 2$ measures their difference (a deviator), $H_{\mathrm{m}}=\left(C_{\mathrm{m} 1}+C_{\mathrm{m} 2}\right) / 2$ is the mean of the principal intrinsic curvatures of the protein $C_{1 \mathrm{~m}}$ and $C_{2 \mathrm{~m}}$, and $\Delta H_{\mathrm{m}}$ $=\left(C_{\mathrm{m} 1}-C_{\mathrm{m} 2}\right) / 2$ measures their difference; $\kappa$ and $\kappa^{*}$ are two independent interaction constants. The mutual orientation of the coordinate systems of the principal curvatures of the membrane and of the protein is defined by the angle $\omega$.

The lateral distribution of proteins can be obtained by minimizing the sum of the free energies for the tether compartment (denoted by index " $t$ ") of radius $R_{t}$ and the GUV, which can be assumed to be practically flat with $H \approx 0$. For the tether $C_{1}=1 / R_{t}$ and $C_{2}=0$, thus $H_{t}=1 /\left(2 R_{t}\right)$. A representative 
simple model for the vesicle is a flat surface with $H_{r}=$ 0 (denoted by index " $r$ "), with a very large area occupied by proteins of a constant density serving as the reservoir from which the thin tether is pulled out and kept at a constant length so that its area is relatively small (Fig. 1b). The free energy relevant for the treated system involves the interaction energies for the two compartments $N_{\mathrm{p}, i} E_{i}$ (with $i=r$ or $t, N_{\mathrm{p}, i}$ the number of proteins and $E_{i}$ defined by eqn (1)) and the corresponding contributions due to the entropy of mixing proteins and lipids. We approximate the latter contributions by a free energy term due to the entropy of the proteins

$$
F_{\mathrm{p}, i}=k_{\mathrm{B}} T N_{\mathrm{p}, i}\left(\ln \frac{N_{\mathrm{p}, i}}{A_{i}-2 N_{\mathrm{p}, i} a_{\mathrm{p}}}-1\right)
$$

where $A_{i}$ is the area of the $i$-th compartment equal to $N_{\mathrm{p}, i} a_{\mathrm{p}}+$ $N_{\mathrm{L}, i} a_{\mathrm{L}}$ with $a_{\mathrm{p}}$ the area of the protein, $a_{\mathrm{L}}$ the area of the lipid molecule and $N_{\mathrm{L}, i}$ the number of lipids in the $i$-th compartment. The free energy (eqn (2)) corresponds to a two-dimensional gas of finite size particles. The inaccessible area of proteins is approximated by the term $2 N_{\mathrm{p}, i} a_{\mathrm{p}}$, i.e., twice the area of proteins, which accounts well for proteins in their dilute solutions. ${ }^{19}$ This formulation does not include direct protein-protein interaction. The solvent contribution to the mixing entropy is neglected. Exact calculations of the entropy contributions to the free energy of the treated system are not within the scope of this analysis. Such treatment can be found in Mahata and Das. ${ }^{20}$ Similar other non-ideal gas models for adsorbed proteins can also demonstrate the sensitivity to vesicle protein concentration..$^{6-8,15}$

\section{(ii) Unidirectional transmembrane protein orientation - small protein density}

We first treat the system in which all inclusions have the same transmembrane orientation. The behavior of this system is governed essentially by six parameters ( $c f$. eqn (1) and (2)), the four parameters of the interaction term $\left(H_{\mathrm{m}}, \Delta H_{\mathrm{m}}, \kappa, \kappa^{*}\right)$, the area of the protein $\left(a_{\mathrm{p}}\right)$ and the reservoir protein number density $\left(m_{\mathrm{p}, r}=N_{\mathrm{p}, r} / A_{r}\right)$ (density in the following). It turned out that it is possible to unravel how the parameters appearing in the interaction term (eqn (1)) relate to different characteristic features of the system's behavior by determining the sorting ratio, i.e., the ratio between the protein densities in the compartments " $t$ " and " $r$ " (denoted by $\hat{S}), \dagger$ for dilute protein solution (or by taking $a_{\mathrm{p}}=0$ ). The entropy term eqn (2) reduces in this limit to the entropy term of the ideal gas. The sorting ratio $\hat{S}$ is then obtained from the requirement of the equality of protein chemical potentials in the tether and the reservoir and is, according to eqn (8) in Kralj-Iglič and coworkers, ${ }^{18}$ equal to

$$
\hat{S}=\frac{q_{\mathrm{c}, t}}{q_{\mathrm{c}, r}} I_{0}\left(\frac{\kappa^{*}}{k_{\mathrm{B}} T} \Delta H_{t} \Delta H_{\mathrm{m}}\right),
$$

\footnotetext{
$\dagger$ This notion $(\hat{S})$ is chosen here to distinguish the defined ratio between the protein areal densities in the tether and flat GUV from the defined sorting ratio $S$ by Aimon and coworkers, ${ }^{5}$ measuring the ratio between the amount of protein per lipid in both compartments.
}

where

$$
q_{\mathrm{c}, \mathrm{i}}=\exp \left(-\frac{\kappa}{2 k_{\mathrm{B}} T}\left(H_{\mathrm{i}}-H_{\mathrm{m}}\right)^{2}-\frac{\kappa^{*}}{2 k_{\mathrm{B}} T}\left(\Delta H_{i}^{2}+\Delta H_{\mathrm{m}}^{2}\right)\right) .
$$

The modified Bessel function of the first kind and of order zero $I_{0}\left(\frac{\kappa^{*}}{k_{\mathrm{B}} T} \Delta H_{t} \Delta H_{\mathrm{m}}\right)$ appears in eqn (3) as a result of averaging in the tether compartment over all possible angles $\omega^{\mathbf{1 8 , 2 1}}$ In eqn (3), it is also taken into account that the corresponding value of this modified Bessel function for the reservoir is unity because of $\Delta H_{r}=0$. By inserting $H_{t}=\Delta H_{t}=1 /\left(2 R_{t}\right)$ into eqn (3) and (4), we obtain for the protein density ratio

$$
\hat{S}=I_{0}\left(\frac{\kappa^{*}}{2 k_{\mathrm{B}} T} \frac{\Delta H_{\mathrm{m}}}{R_{t}}\right) \exp \left(-\frac{\kappa+\kappa^{*}}{8 k_{\mathrm{B}} T} \frac{1}{R_{t}^{2}}+\frac{\kappa}{2 k_{\mathrm{B}} T} \frac{H_{\mathrm{m}}}{R_{t}}\right) .
$$

We first show that the function describing the dependence of the sorting ratio $\hat{S}$ on the tether curvature $1 / R_{t}$ depends only on two independent constants related to the protein mean curvature and the protein anisotropy. By conveniently defining these constants to be directly related to the protein properties $H_{\mathrm{m}}$ and $\Delta H_{\mathrm{m}}$ as

$$
\alpha=\frac{\kappa}{\left(k_{\mathrm{B}} T\left(\kappa+\kappa^{*}\right)\right)^{\frac{1}{2}}} H_{\mathrm{m}}
$$

and

$$
\beta=\frac{\kappa^{*}}{\left(k_{\mathrm{B}} T\left(\kappa+\kappa^{*}\right)\right)^{\frac{1}{2}}} \Delta H_{\mathrm{m}},
$$

the function $\hat{S}$ reads

$$
\hat{S}=I_{0}(\beta t) \exp \left(-\frac{t^{2}}{2}+\alpha t\right)
$$

with the new variable $t$ being a measure of membrane curvature of the tether

$$
t=\left(\frac{\kappa+\kappa^{*}}{4 k_{\mathrm{B}} T}\right)^{\frac{1}{2}} \frac{1}{R_{t}}
$$

Function $\hat{S}$ exhibits a maximum at $t=t_{\mathrm{e}}$, which is the solution of the equation obtained by requiring that the derivative of $\hat{S}$ defined by eqn (8) by $t$ equals zero,

$$
\beta I_{1}\left(\beta t_{\mathrm{e}}\right)+I_{0}\left(\beta t_{\mathrm{e}}\right)\left(-t_{\mathrm{e}}+\alpha\right)=0 .
$$

In eqn (10), $I_{1}\left(\beta t_{\mathrm{e}}\right)$ is the modified Bessel function of the first kind and order one. The maximum sorting ratio $\hat{S}_{\mathrm{e}}$ is obtained by inserting $t_{\mathrm{e}}$ into eqn (8). Note that $\hat{S}_{\mathrm{e}}$ is a measurable quantity. Thus, it is of interest to investigate the combinations of the constants $\alpha$ and $\beta$ for which a given measured value of $\hat{S}_{\mathrm{e}}$ is achieved. Note also that $\alpha$ is the scaled protein mean curvature and $\beta$ is a measure of anisotropy in protein shape. Fig. 2 shows the dependence of the ratio $\hat{S}$ on $t / t_{\mathrm{e}}$ for three combinations of constants $\alpha$ and $\beta$, which yield the same maximum sorting ratio $\hat{S}_{\text {e }}$ The three sorting ratio curves differ in their initial slope. The 


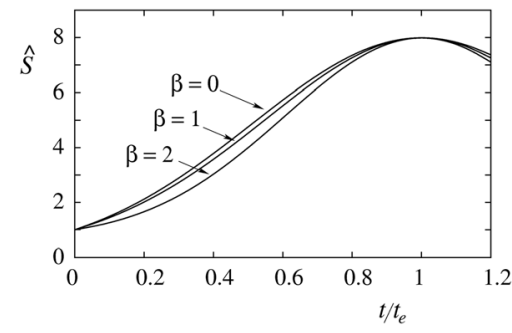

Fig. 2 Dependence of the sorting ratio $(\hat{S})$ on the reduced curvature $\left(t / t_{\mathrm{e}}\right)$ in the limit of low protein density $\left(m_{\mathrm{p}, \mathrm{r}} \approx 0\right)$ for $\hat{S}_{\mathrm{e}}=8$ and $\beta=0,1$, and 2 . The corresponding values of the constant $\alpha$ are 2.04, 1.60 and 0.76 .

larger is the degree of protein anisotropy expressed by the value of the constant $\beta$, the smaller is the initial slope. Thus, at tether curvatures different from $t_{\mathrm{e}}$ (the point of maximum $\hat{S}$ ) the sorting of anisotropic proteins is smaller than that of the isotropic ones.

It is instructive already in the limit of low protein density ( $m_{\mathrm{p}, \mathrm{r}} \approx 0$ ) to evaluate the relation between the constants $\alpha$ and $\beta$ and measurable properties of the sorting ratio curve $\hat{S}_{\mathrm{e}}$. When $\beta=0$, we have (see eqn (10)) $t_{\mathrm{e}, 0}=\alpha_{0}$, which gives $\hat{S}_{\mathrm{e}, 0}=\exp \left(\frac{\alpha_{0}^{2}}{2}\right)$. When $\beta \neq 0$ the same sorting ratio $\hat{S}_{\mathrm{e}, 0}$ can be reached at smaller values of the constant $\alpha$ (Fig. 3a), whereas the scaled tether curvature $t_{\mathrm{e}}$ for which the maximal $\hat{S}$ is realized shifts to larger values (Fig. $3 \mathrm{~b})$. The slope of the $\hat{S}\left(t / t_{\mathrm{e}}\right)$ curve at small values of tether curvature, which at $\beta=0$ is equal to $\alpha_{0}$, decreases with $\beta$ (Fig. 2).

\section{(iii) Unidirectional transmembrane protein orientation - finite protein density}

The sorting ratio $\hat{S}$ (eqn (5)) obtained in the limit of low protein density $\left(m_{\mathrm{p}, r} \approx 0\right)$ does not depend on the protein density on the
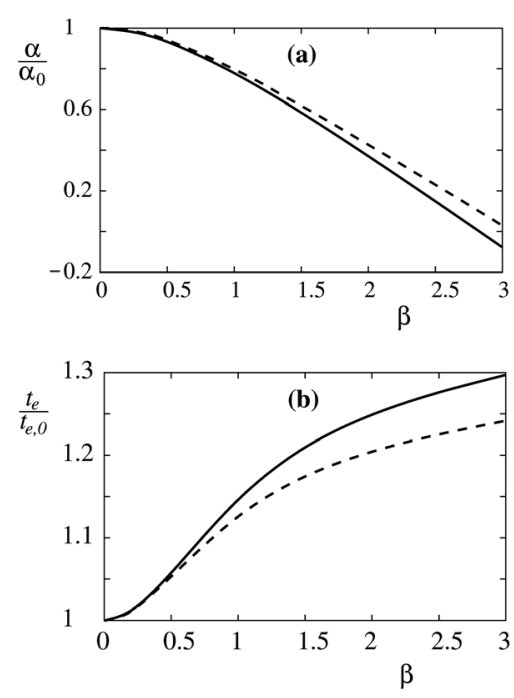

Fig. 3 Relations between the protein mean curvature constant $(\alpha)$ and the protein anisotropy constant $(\beta)$, and the curvature at which a given maximum sorting ratio is reached $\left(t_{\mathrm{e}}\right)$ at a given maximum value of the sorting ratio in the limit of low protein density $\left(m_{\mathrm{p}, \mathrm{r}} \approx 0\right)$. The effects of $\beta$ (a) on $\alpha$, and (b) on $t_{\mathrm{e}}$ for $\hat{S}_{\mathrm{e}}=8$ (solid line) and 15 (dashed line) are shown relative to the values of isotropic inclusions. flat vesicle region (reservoir) and therefore does not represent the observed behavior, which shows the diminishing of $\hat{S}\left(1 / R_{t}\right)$ at the higher protein density. ${ }^{5}$ Such behavior can be ascribed to finite protein sizes, which affect the system through the entropy contribution to its free energy as considered here by eqn (2). The corresponding generalization of the equilibrium condition (eqn (8)) obtained by the requirement of the equality of protein chemical potential in the reservoir and the tether can be expressed in terms of the protein concentration of the reservoir $\left(m_{\mathrm{p}, r}\right)$ and the density ratio as

$$
f(\hat{S} ; \gamma) \hat{S}=I_{0}(\beta t) \exp \left(-\frac{t^{2}}{2}+\alpha t\right),
$$

where the function $f(\hat{S} ; \gamma)$ is

$$
f(\hat{S} ; \gamma)=\frac{1-\gamma}{1-\gamma \hat{S}} \exp \left(\frac{\gamma \hat{S}}{1-\gamma \hat{S}}-\frac{\gamma}{1-\gamma}\right)
$$

with the new unit-less constant $\gamma$, introduced for the product $2 a_{\mathrm{p}} m_{\mathrm{p}, r}$. The appearance of this product, and thus $\gamma$, is due to the incorporation of finite protein size in our analysis, and it accounts for the excluded area of the proteins in the reservoir. It should be noted that $f(1 ; \gamma)=1$ and that $f(\hat{S} ; \gamma)$ is a monotonically increasing function of $\hat{S}$ (Fig. 4). Derivative of the product $f(\hat{S} ; \gamma) \hat{S}$ on the left side of eqn (11) is thus always positive and $f(\hat{S} ; \gamma)$ is larger than one. Therefore, the product $f(\hat{S} ; \gamma) \hat{S}$ has its maximum at the same $t_{\mathrm{e}}$ as that obtained from eqn (10). However, $\hat{S}_{\mathrm{e}}$ is lower by the factor $f\left(\hat{S}_{\mathrm{e}} ; \gamma\right)$. It is to be noted that the more comprehensive treatment of the area exclusion effects for the entropic contribution to free energy will not change the qualitative behavior of $f(\hat{S} ; \gamma)$ from what we demonstrate here.

The area exclusion constant $\gamma$ has an effect on the dependence of $\hat{S}$ on curvature. In Fig. 5 it is shown by two representative examples how it affects this dependence by keeping the maximum sorting ratio $\hat{S}_{\mathrm{e}}$ constant, and appropriately adjusting constants $\alpha$ and $\beta$. The protein in Fig. $5 \mathrm{a}$ is considered to be isotropic $(\beta=0)$ and the protein in Fig. $5 \mathrm{~b}$ to be anisotropic (with $\alpha=\beta$ ). It can be seen that at finite values of the constant $\gamma$, the curves are running above the curve at $\gamma=0$. The effect of $\gamma$ in isotropic and anisotropic cases is practically similar. This can also be inferred from the appearance of $\gamma$ only on the left hand side of eqn (11).

The $\hat{S}\left(1 / R_{t}\right)$ curves measured at different protein densities also provide information about the corresponding values of the constants $\alpha, \beta$ and $\gamma$. This will be illustrated by taking into

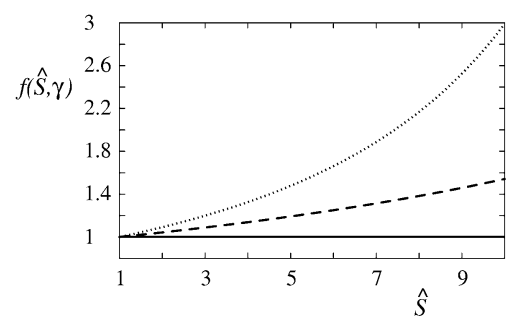

Fig. 4 Function $f(\hat{S} ; \gamma)$ for values of the area exclusion constant $\gamma=$ 0 (solid line), 0.02 (dashed line), and 0.04 (dotted line). 

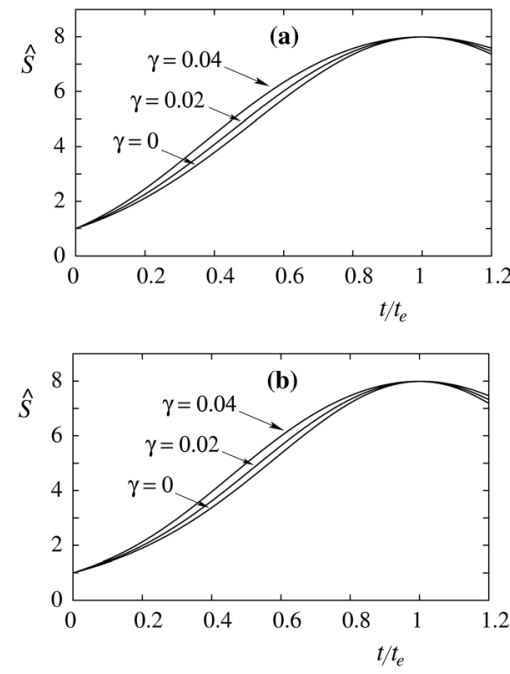

Fig. 5 Dependence of the sorting ratio $\hat{S}$ on the reduced curvature $t / t_{\mathrm{e}}$ for the indicated values of the area exclusion constant $\gamma(0,0.02$ and $0.04)$. (a) The case of an isotropic protein $(\beta=0)$. The respective values of the constant $\alpha$ are 2.04, 2.19 and 2.39. (b) The case of an anisotropic protein (with $\alpha=\beta$ ). The respective values of the constant $\alpha=\beta$ are $1.34,1.41$ and 1.50

consideration that these curves are measured at two reservoir protein densities as it was presented by Aimon and coworkers. ${ }^{5}$ The available data in this case include the two maximum sorting ratios, $\hat{S}_{\text {e,high }}$ for the higher and $\hat{S}_{\text {e,low }}$ for the lower reservoir protein density, and the ratio between the reservoir densities $m_{\mathrm{p}, r, \text { high }} / m_{\mathrm{p}, r, \text { low }}$. Because the constant $\gamma$ is proportional to the reservoir density, the latter ratio also gives us the ratio $\gamma_{\text {high/ }}$ $\gamma_{\text {low. }}$ From eqn (11) it follows that for given values of the constants $\alpha$ and $\beta$ we have

$$
f\left(\hat{S}_{\mathrm{e}, \text { low }} ; \gamma_{\text {low }}\right) \hat{S}_{\mathrm{e}, \text { low }}=f\left(\hat{S}_{\mathrm{e}, \text { high }} ; \gamma_{\text {high }}\right) \hat{S}_{\mathrm{e}, \text { high }} .
$$

Eqn (13) can be used, by choosing $\hat{S}_{\text {ellow }}$ and $\gamma_{\text {high }} / \gamma_{\text {low }}$, to determine which value of $\gamma_{\text {low }}$ corresponds to a given value of the ratio $\hat{S}_{\text {e,high }} / \hat{S}_{\text {ellow }}$. The corresponding dependence is presented in Fig. 6a for $\hat{S}_{\text {ellow }}=8$ and $\gamma_{\text {high }} / \gamma_{\text {low }}=10$. From the measured value of $\gamma_{\text {low }}$, it is then possible by the use of eqn (11) and (12), or equivalently Fig. 6a, to extract the values of the constants $\alpha$ and $\beta$ analogously to the corresponding description in the subsection (ii). As an example, we take $\beta=0$. Then, from the theoretical $\hat{S}_{\mathrm{e}}(\gamma)$ curve and known $\hat{S}_{\mathrm{e}}\left(\gamma_{\text {low }}\right)$ we obtain the sorting ratio at $\gamma=0\left(\hat{S}_{\mathrm{e}, 0}\right)$ and thus the constant $\alpha$ from eqn (8). To illustrate this, we plot in Fig. $6 \mathrm{~b} \hat{S}_{\mathrm{e}}(\gamma)$ for different values of $\hat{S}_{\mathrm{e}, 0}$. The sought $\hat{S}_{\mathrm{e}, 0}$ corresponds to the curve which intersects the dotted $\hat{S}_{\mathrm{e}}=8$ line at $\gamma=\gamma_{\text {low }}$.

\section{(iv) Bidirectional orientation of transmembrane proteins}

In previous subsections, we have discussed the case of a single integral membrane protein species whose molecules are oriented across the membrane in one direction only. That is, they induce curvature of one sign only (either positive or negative). In this subsection, we will look into the case when
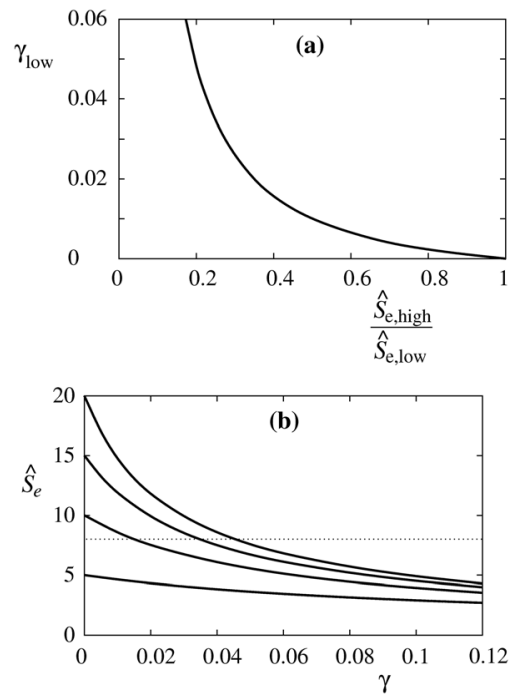

Fig. 6 Description of a possible way to determine the constant $\gamma_{\text {low }}$ from the maximum sorting ratios at two different reservoir protein densities $m_{\mathrm{p}, \mathrm{r}}$. In the treated example $\beta=0$. (a) Dependence of $\gamma_{\text {low }}$ on the ratios $\hat{S}_{e, \text { high }} / \hat{S}_{\mathrm{e} \text {,low }}$ for $\hat{S}_{\mathrm{e} \text {,low }}=8$ and $m_{\mathrm{p}, \mathrm{r} \text {, high }} / m_{\mathrm{p}, \mathrm{r} \text {, low }}=10$. (b) Dependence of the maximum sorting ratio $\hat{S}_{e}$ on the area exclusion constant $\gamma$ for $\hat{S}_{\mathrm{e}, 0}=5,10,15$ and 20 . The dotted line corresponds to $\hat{S}_{\text {ellow }}=8$.

molecules of the transmembrane protein are oriented in both ways. We will consider them as two different species ("+" and “-”). We take that in the GUV (or flat membrane) the densities of proteins with positive and negative orientation are ${m_{\mathrm{p}, r}}^{+}=$ $\nu m_{\mathrm{p}, r}$ and $m_{\mathrm{p}, r^{-}}=(1-\nu) m_{\mathrm{p}, r}$, respectively, so that $m_{\mathrm{p}, r}=m_{\mathrm{p}, r}{ }^{+}+$ $m_{\mathrm{p}, r}{ }^{-}$, where $\nu$ is the fraction of protein molecules of positive orientation. Then, $\nu=1$ implies GUV has proteins with positive orientation only (see subsections (ii) and (iii)), and $\nu=0$ denotes that all the proteins present have negative orientation. In the corresponding interaction terms (cf. eqn (1)), both the intrinsic mean and deviatoric curvatures of these species will have the same magnitude, but they will differ by a sign $\left(H_{\mathrm{m}}{ }^{+}=-H_{\mathrm{m}}\right.$ and $\Delta H_{\mathrm{m}}{ }^{+}=-\Delta H_{\mathrm{m}}{ }^{-}$). Furthermore, the proteins have the same area $a_{\mathrm{p}}$. As a result we have $\alpha^{+}=-\alpha^{-}=\alpha$, and $\beta^{+}=-\beta^{-}=\beta$. The entropic contribution to free energy given by eqn (2) gets modified into

$$
F_{\mathrm{p}, i}{ }^{ \pm}=k_{\mathrm{B}} T N_{\mathrm{p}, i}{ }^{ \pm}\left(\ln \frac{N_{\mathrm{p}, i}{ }^{ \pm}}{A_{i}-\sum 2 N_{\mathrm{p}, i}{ }^{ \pm} a_{\mathrm{p}}}-1\right),
$$

where the summation is performed over both protein species.

In analogy, with the case of protein molecules having the same transmembrane orientation, the free energy of this more general system has to be minimized with respect to the equilibrium distribution between the tether and flat membrane compartments of both species. The equilibrium equations analogous to eqn (11) are then

$$
f(\hat{S} ; \gamma) \frac{m_{\mathrm{p}, t}{ }^{+}}{m_{\mathrm{p}, r^{+}}}=I_{0}(\beta t) \exp \left(-\frac{t^{2}}{2}+\alpha t\right)
$$


and

$$
f(\hat{S} ; \gamma) \frac{m_{\mathrm{p}, t^{-}}}{m_{\mathrm{p}, r^{-}}}=I_{0}(\beta t) \exp \left(-\frac{t^{2}}{2}-\alpha t\right)
$$

from which we get

$$
f(\hat{S} ; \gamma) \hat{S}=I_{0}(\beta t) \exp \left(-\frac{t^{2}}{2}\right)(\nu \exp (\alpha t)+(1-\nu) \exp (-\alpha t)) .
$$

From eqn (17), it can be concluded that bidirectional orientation affects the behavior of the system at the level of the interaction term. Therefore, it is sufficient to investigate its consequences in the limit of dilute proteins when $f(\hat{S} ; \gamma)=1$. As an example of the basic behavior of the system due to the bidirectional orientation, we present in Fig. 7a the sorting of $\hat{S}_{+}=m_{\mathrm{p}, t}{ }^{+} / m_{\mathrm{p}, r}$ and $\hat{S}_{-}=m_{\mathrm{p}, t}{ }^{-} / m_{\mathrm{p}, r}$ of the positively and negatively oriented proteins, respectively, for $\nu=0.5$ and $\beta=1$. Here, $\nu=0.5$ implies that initially in the flat membrane the two types of proteins have been present in equal amounts. With increasing curvature (positive) of the tether, we observe that the proteins with positive orientation enrich in the tether and the negatively orientated proteins get depleted from the tether. The same has also been observed for other values of $\nu$ as well. The behavior for $\nu=0.5$ has also been demonstrated by Aimon and coworkers. ${ }^{5}$ We also present the sorting for different values of the fraction $\nu$ in Fig. $7 \mathrm{~b}-\mathrm{d}$ for $\beta=0,1$, and 2, respectively. Decreasing values of $\nu$ imply increasing fraction of protein with negative orientation. The results and the scenario for $\nu=1$ are the same as that considered for the proteins with the same orientation, presented in subsections (ii) and (iii) and Fig. 2. We observe that the behavior of the sorting ratio curve for different $\beta$ is similar for all values of $\nu$. Moreover, with increasing $\beta$ the effect of $\nu$ diminishes and with decreasing $\nu$ the effect of $\beta$ on sorting reduces.
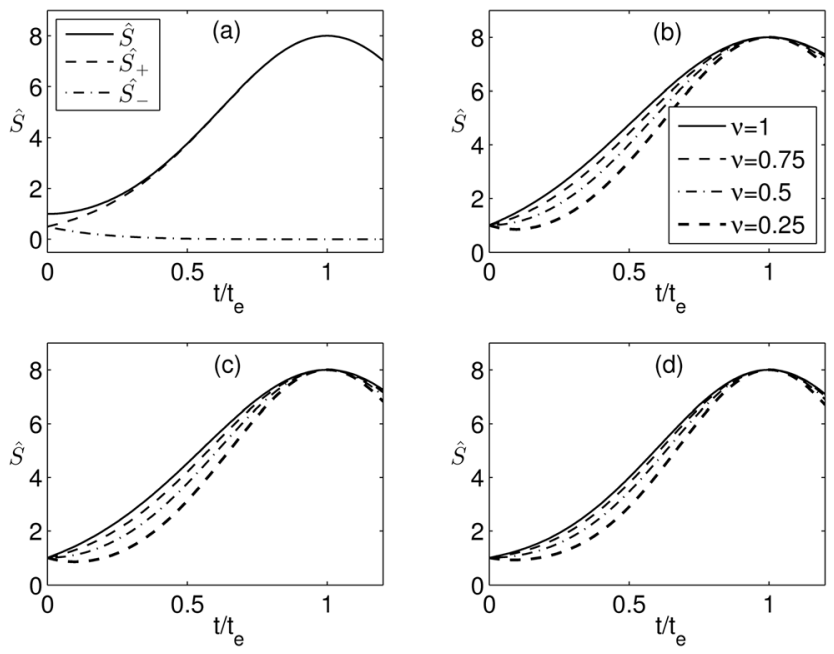

Fig. 7 Dependence of the sorting ratio $\hat{S}$ on the reduced curvature $t / t_{\mathrm{e}}$ for $\hat{S}_{\mathrm{e}}=8$. (a) For $\beta=1$ and $\nu=0.5 . \hat{S}_{+}$and $\hat{S}_{-}$are the sorting ratios of the positively and negatively oriented proteins, respectively. (b)-(d) For $\beta=0,1$, and 2, respectively, and four different values of $\nu$ as shown.

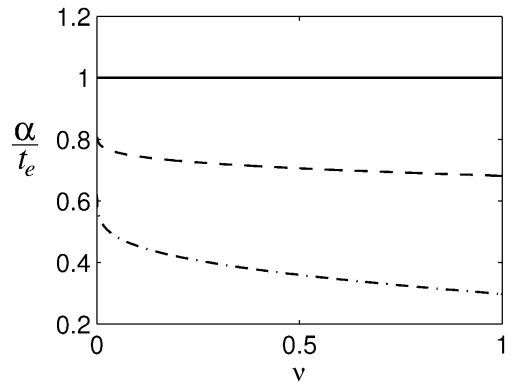

Fig. 8 Dependence of the ratio $\alpha / t_{\mathrm{e}}$ corresponding to $\hat{S}_{\mathrm{e}}=8$ on $\nu$ for $\beta$ $=0$ (solid line), 1 (dashed line), and 2 (dash-dot line).

The values of the scaled mean intrinsic curvature $\alpha$ of proteins and scaled tether radius $t_{\mathrm{e}}$ corresponding to the maximum sorting ratio also depend on the fraction $\nu$. We demonstrate that $\alpha$ decreases and $t_{\mathrm{e}}$ increases with $\beta$ for all values of $\nu$ considered, which is consistent with Fig. 3a and $\mathrm{b}$ for $\nu=1$. In Fig. 8, we also present the variation of the ratio $\alpha / t_{\mathrm{e}}$ with $\nu$. For $\beta=0$ the ratio $\alpha / t_{\mathrm{e}}$ is almost (but not exactly) equal to one for all $\nu$. For nonzero $\beta$, the ratio decreases with $\nu$. This implies that the tether curvature required to attain a specified maximum sorting is larger. Further, both $\alpha$ and $t_{\mathrm{e}}$ decrease with $\nu$ (that is with enrichment of proteins of positive orientation in the GUV). When only the proteins with negative orientation are present in the flat membrane (that is $\nu=0$ ), the density ratio $\hat{S}$ decreases with tether curvature. However, if the membrane with negative curvature is present, enrichment occurs, and for maximum sorting, the tether curvature $t_{\mathrm{e}}$ is negative.

\section{(v) Determination of the parameters of the protein-lipid bilayer interaction}

The next step of the analysis is to extract from the values of the constants $\alpha, \beta$ and $\gamma$, all available information about the parameters of the interaction term, eqn (1). The expressions for the mean protein principal curvature and the difference between protein principal curvatures are obtained by dividing eqn (6) and (7), respectively, by eqn (9) for $t=t_{\mathrm{e}}$, and denoting the tether radius at the maximum sorting as $R_{t, \mathrm{e}}$. The obtained expressions are

$$
R_{t, \mathrm{e}} H_{\mathrm{m}}=\frac{\kappa+\kappa^{*}}{2 \kappa} \frac{\alpha}{t_{\mathrm{e}}}
$$

and

$$
R_{t, \mathrm{e}} \Delta H_{\mathrm{m}}=\frac{\kappa+\kappa^{*}}{2 \kappa^{*}} \frac{\beta}{t_{\mathrm{e}}}
$$

The structural features of the protein, $H_{\mathrm{m}}$ and $\Delta H_{\mathrm{m}}$, in eqn (18) and (19) are related to the experimentally obtained value of $R_{t, \mathrm{e}}$ and from the above fit values of $t_{\mathrm{e}}, \alpha$ and $\beta$. However, the interaction term eqn (1) involves more parameters than can be extracted from the $\hat{S}\left(t / t_{\mathrm{e}}\right)$ curves. In eqn (18) and (19), the unknown parameter is the ratio $\kappa^{*} / \kappa$. It can be concluded that it is possible to obtain by the described procedure only the combinations of $H_{\mathrm{m}}$ and $\Delta H_{\mathrm{m}}$ that give the same $\hat{S}\left(t / t_{\mathrm{e}}\right)$ dependence. 
It is noteworthy that the curvature at which the ratio $\hat{S}$ attains the maximum does not depend on the strength of the proteinlipid bilayer interaction expressed by the parameters $\kappa$ and $\kappa^{*}$. These parameters instead affect the magnitude of $\hat{S}_{\mathrm{e}}$.

\section{Discussion}

Biological membranes are lipid based but crowded by many different proteins and protein complexes serving different functions, among which are the establishment of membrane curvature and its sensing. Effects of proteins on the latter can be treated theoretically at various structural levels, from molecular or atomistic to macroscopic. Here, we pursued the macroscopic approach by using a set of phenomenological parameters that define the protein-lipid bilayer interaction caused by the mismatch between the intrinsic principal curvatures of the protein and the principal curvatures of membrane. Proteins were assumed to be rigid. The crucial parameters of the corresponding interaction term (eqn (1)) are the principal curvatures of the shape of the protein that is in contact with the surrounding lipid molecules. These curvatures are in a mismatch with the principal curvatures $C_{1}$ and $C_{2}$ that a membrane would have at the position of a protein in its absence. This formulation implies that while due to a protein the curvature of the surrounding membrane is modified, this modification decays into the bulk membrane curvatures $C_{1}$ and $C_{2}$ within a relatively short characteristic distance. The validity of the approach thus depends on how much is this characteristic distance smaller than the average closest distance between proteins.

The effects of protein shape have also been considered by other researchers, who assumed that it modifies membrane local spontaneous curvature. The predictions of the spontaneous curvature and protein-lipid bilayer interaction term approaches look similar; however, they differ in details. In the limit of weak interaction constant, the model based on the interaction term reduces ${ }^{\mathbf{1 3}}$ into the spontaneous curvature model; therefore, it can be considered to be more general. The qualitative difference between the two approaches comes to light particularly at small protein concentrations. Due to the curvature dependent interaction term the curvature differences are sensed even by a single protein, whereas in the spontaneous curvature approach the membrane curvature must first be formed, which requires the presence of a considerable number of molecules, and only then the curvature is felt by the additional proteins. In the tether pulling experiment, as will be demonstrated later, this feature is reflected in the initial slope of the sorting curve.

The general analysis on which parameters of the treated protein-lipid bilayer interaction term can be extracted from measured dependence of the sorting ratio on tether curvature showed the system behavior to be defined by the protein mean curvature constant $\alpha$ and the protein anisotropy constant $\beta$ based on the parameters of the interaction term (eqn (1)) and the area exclusion constant $\gamma$ arising from the entropy contribution to the system's free energy. The first two constants are related to the mean protein intrinsic principal curvature and to the difference between protein intrinsic principal curvatures, respectively.

We analyzed the dependence of the sorting ratio on the constants $\alpha, \beta$ and $\gamma$. The main feature of the sorting ratio curve is that there exists a tether radius at which this ratio is maximal. This implies that at the maximal sorting, the mismatch between the tether curvature and the protein intrinsic curvatures is least. The same maximum sorting ratio can be attained by many combinations of the constants $\alpha, \beta$ and $\gamma$, appearing at different tether radii. At a specified tether radius, the sorting ratio of isotropic protein is higher than that in the case of anisotropic protein. Further, the tether radii at which the sorting ratio is maximal are determined only by the constants $\alpha$ and $\beta$ whereas the maximum sorting ratio depends also on the constant $\gamma$. As an important indicator of the protein isotropy features, we identified the slope of the sorting ratio curve at small tether curvature, which for more anisotropic proteins is lower (Fig. 2). This initial slope does not depend on the constant $\gamma$ (Fig. 5). Consequently, the effect of the area exclusion constant $\gamma$ on the sorting ratio curve of isotropic and anisotropic proteins is almost the same (Fig. 5). It is also notable that the bidirectional orientation effect of the proteins is manifested at the level of the protein-phospholipid membrane interaction term only (eqn (17)).

The above general conclusions will be illustrated by the analysis of the expression for the protein-lipid bilayer interaction $^{18}$ in view of recent experiments exploring the effect of membrane curvature on the distribution of proteins over the variably curved vesicle membrane. ${ }^{5}$ X-ray structural studies of the open state of KvAP show ${ }^{22}$ that this tetrameric channel is 4fold symmetric, which indicates that $\beta=0$. It is possible, from the experimental value of $\hat{S}_{\mathrm{e}}$ at two protein densities and the $\hat{S}(t)$ $t_{\mathrm{e}}$ ) dependence, to obtain reasonable values of the remaining parameters $\alpha$ and $\gamma$. The measured points of the KvAP sorting ratio, denoted by Aimon and coworkers ${ }^{5}$ as $S$, we transformed into the true KvAP sorting ratio $(\hat{S})$ by the relationship

$$
\hat{S}=\frac{S}{1+m_{\mathrm{p}, r} a_{\mathrm{p}}(S-1)} .
$$

From Fig. 9 it can be seen that the experimental points at the lower density of KvAP in the vesicle can be well approximated with the curve obtained by taking $\alpha=2.15$ and $\gamma=0.015$. By taking $a_{\mathrm{p}}=45 \mathrm{~nm}^{2}$ and $m_{\mathrm{p} \text {,low }}=162 \mu \mathrm{m}^{-2}$ we get $\gamma=0.0146$, which is in view of quoted experimental errors surprisingly close to the value obtained through the analysis. Furthermore, it can be noted, at least on the less scattered low concentration $S\left(1 / R_{t}\right)$ curve (Fig. 2 of Aimon and coworkers ${ }^{5}$ ) that the initial slope is finite, which supports the treatment based on the interaction term.

Through eqn (18) and (19), the interaction parameters $\kappa$ and $\kappa^{*}$, and the protein shape determinants $H_{\mathrm{m}}$ and $\Delta H_{\mathrm{m}}$, are related to the constants $\alpha$ and $\beta$. However, the number of parameters of the interaction term $\left(\kappa, \kappa^{*}, H_{\mathrm{m}}, \Delta H_{\mathrm{m}}\right)$ is larger than the number of constants that determine the dependence of the sorting ratio on the tether curvature $(\alpha$ and $\beta$ ). Consequently, by measuring only this dependence the interaction parameters are undeterminable. It is nevertheless possible to 


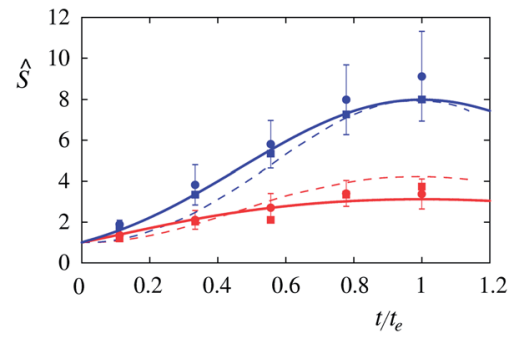

Fig. 9 Comparison of the predictions of the presented model for the values of the area exclusion constant $\gamma=0.015$ (upper curve) and $\gamma=$ 0.15 (lower curve) and constant $\alpha=2.15$ with the experimentally measured dependence of the sorting ratio $\hat{S}$ on tether curvature for potassium channel KvAP obtained by Aimon and coworkers. ${ }^{5}$ The experimental points (the mean values, the median values and the standard deviations) are from the work of Aimon and coworkers ${ }^{5}$ and recalculated according to eqn (20), by taking $m_{\mathrm{p}, r \text {, low }}=162 \mu \mathrm{m}^{-2}$, $m_{\mathrm{p}, r \text {, high }}=1777 \mu \mathrm{m}^{-2}$ and $a_{\mathrm{p}}=45 \mathrm{~nm}^{2}$. Dashed lines represent the fit obtained by the model developed by Aimon and coworkers. ${ }^{5}$

make plausible estimates. For example, by assuming $\kappa=\kappa^{*}$ the principal curvatures of the protein KvAP are predicted (eqn (18)) to be equal to $1 / R_{t, \mathrm{e}}$, which is $\approx 0.1 \mathrm{~nm}^{-1}$. This value corresponds to the radius of curvature of the slope in the paddle shape of KvAP presented in Fig. 4E of Cuello and collaborators (also reproduced in Fig. 10). ${ }^{23}$ Within the indeterminacy of the ratio $\kappa^{*} / \kappa$, the intrinsic principal curvature of the isotropic protein KvAP obtained by measuring the sorting ratio was found to be consistent with the X-ray diffraction obtained shape of one of this protein's conformations. Corresponding intrinsic spontaneous curvature of this protein $\left(c_{\mathrm{p}}=2 \mathrm{H}_{\mathrm{m}}\right)$ is thus predicted to be $1 / 5 \mathrm{~nm}^{-1}$, which is five times higher than the value presented by Aimon and collaborators. ${ }^{5}$

As it was demonstrated, by the analysis of the observed dependence of the sorting ratio on the tether curvature, it is possible to extract a few model parameters. These parameters could then be used to obtain a consistent picture about the functioning of a studied membrane protein in conjunction with other structural level studies. For example, the microscopic theories could within the context of this study contribute by estimating the ratio $\kappa^{*} / \kappa$, whereas structural studies could provide an estimate of protein principal curvatures.

The present analysis was based on the protein-lipid bilayer interaction and assuming proteins to be rigid. The treatment of proteins as rigid entities is from the point of view of a systematic

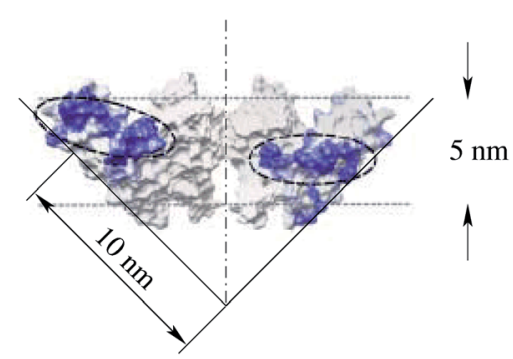

Fig. 10 Estimate of the intrinsic principal curvature of the paddle shape of KvAP as presented in Fig. 4E of Cuello and collaborators. ${ }^{23}$ development of a general model an approximation. However, it can be considered as a useful intermediate step also in situations when it is necessary to take into account protein internal degrees of freedom. For example, in a protein exhibiting different conformational states with different intrinsic curvatures the present model could be generalized by defining, for each of these states, a separate interaction term. Analogous generalization could also be made for proteins with finite compressibility. Moreover, the attractive/repulsive interaction between proteins and between protein and lipids may play a significant role, in addition to the area exclusion effect considered here, at higher protein concentrations. It will be therefore of interest to extend the studies of membrane curvature effects by including these other features of the system.

\section{Acknowledgements}

This work was supported by the Slovenian research agency through the research program P1-0055 and in part by the Project of Knowledge Innovation program (PKIP) of Chinese Academy of Sciences, grant no. KJCX2.YW.W10. S. L. Das acknowledges financial support from Department of Science and Technology, Government of India, through grant no. DST/ $\mathrm{ME} / 20120003$.

\section{References}

1 C. Mim and V. M. Unger, Trends Biochem. Sci., 2012, 37, 526533.

2 T. Baumgart, B. R. Capraro, C. Zhu and S. L. Das, Annu. Rev. Phys. Chem., 2011, 62, 483-506.

3 R. Phillips, T. Ursell, P. Wiggins and P. Sens, Nature, 2009, 459, 379-385.

4 R. Parthasathy and J. T. Groves, Soft Matter, 2007, 3, 24-33.

5 S. Aimon, A. Callan-Jones, A. Berthaud, M. Pinot, G. E. S. Toombes and P. Bassereau, Dev. Cell, 2014, 28, 212-218.

6 P. Singh, T. Mahata, T. Baumgart and S. L. Das, Phys. Rev. E: Stat., Nonlinear, Soft Matter Phys., 2012, 85, 051906.

7 B. Sorre, A. Callan-Jones, J. Manzi, B. Goud, J. Prost, P. Bassereau and A. Roux, Proc. Natl. Acad. Sci. U. S. A., 2012, 109, 173-178.

8 C. Zhu, S. L. Das and T. Baumgart, Biophys. J., 2012, 102, 1837-1845.

9 J. Zimmerberg and M. M. Kozlov, Nat. Rev. Mol. Cell Biol., 2006, 7, 9-19.

10 V. S. Markin, Biophys. J., 1981, 36, 1-19.

11 S. Svetina, V. Kralj-Iglič and B. Žekšs, in Biophysics of Membrane Transport, ed. J. Kuczera and S. Przestalski, 1990, vol. II, pp. 139-155.

12 V. Kralj-Iglič, S. Svetina and B. Žekš, Eur. Biophys. J., 1996, 24, 311-321.

13 B. Božič, V. Kralj-Iglič and S. Svetina, Phys. Rev. E: Stat., Nonlinear, Soft Matter Phys., 2006, 73, 041915.

14 N. S. Mueller, R. Wedlich-Söldner and F. Spira, Mol. Membr. Biol., 2012, 29, 186-196. 
15 S. Perutková, V. Kralj-Iglič, M. Frank and A. Iglič, J. Biomech., 2010, 43, 1612-1617.

16 H. Cui, C. Mim, F. X. Vázquez, E. Lyman, V. M. Unger and G. A. Voth, Biophys. J., 2013, 104, 404-411.

17 F. X. Vázquez, V. M. Unger and G. A. Voth, Biophys. J., 2013, 104, 396-403.

18 V. Kralj-Iglič, V. Heinrich, S. Svetina and B. Žekš, Eur. Phys. J. $B, 1999,10,5-8$.
19 T. L. Hill, An Introduction to Statistical Thermodynamics, Addison-Wesley Publishing Company, Inc., USA, 1962, ch. 16.

20 P. Mahata and S. L. Das, Phys. Rev. E: Stat., Nonlinear, Soft Matter Phys., 2014, 90, 062713.

21 J. B. Fournier, Phys. Rev. Lett., 1996, 76, 4436-4439.

22 Y. Jiang, A. Lee, J. Chen, V. Ruta, M. Cadene, B. T. Chait and R. MacKinnon, Nature, 2003, 423, 33-41.

23 L. G. Cuello, D. M. Cortes and E. Perozo, Science, 2004, 306, 491-495. 